Editorial

Herz 2019 • 44:189-191

https://doi.org/10.1007/s00059-019-4797-4

(c) Springer Medizin Verlag GmbH, ein Teil von Springer Nature 2019

Check for
updates

N. Marx' $\cdot$ R. Dörrr, ${ }^{2,4}$ D. Tschöpe ${ }^{3,4}$

${ }^{1}$ Medizinische Klinik I - Kardiologie, Angiologie und Internistische Intensivmedizin, Universitätsklinikum Aachen, RWTH Aachen, Aachen, Deutschland

${ }^{2}$ Praxisklinik Herz und Gefäße, Dresden, Deutschland

${ }^{3}$ Diabeteszentrum am Herz- und Diabeteszentrum NRW, Universitätsklinik der Ruhr-Universität Bochum, Bad Oeynhausen, Deutschland

${ }^{4}$ Stiftung „Der herzkranke Diabetiker", Stiftung in der Deutschen Diabetes-Stiftung, Bad Oeynhausen, Deutschland

\title{
Herzinsuffizienz und Diabetes - unterschätzt, wenig verstanden und zu selten diagnostiziert
}

Prävalenz der Herzinsuffizienz bei Patienten mit Prädiabetes und Diabetes extrem hoch sind. Ferner legen neueste Daten nahe, dass Herzinsuffizienz der zweithäufigste Grund für die initiale klinische Präsentation einer kardiovaskulären Erkrankung bei Patienten mit Diabetes ist. Interessanterweise liegt die Herzinsuffizienz damit noch vor der stabilen Angina pectoris oder dem nichttödlichen Myokardinfarkt. Das Vorliegen einer Herzinsuffizienz bei Patienten mit Diabetes führt zu einer deutlich erhöhten Mortalität im Vergleich zu herzinsuffizienten Patienten ohne Diabetes; bis zu 50\% aller Patienten mit Diabetes, die wegen einer kongestiven Herzinsuffizienz hospitalisiert wurden, sterben binnen 3 Jahren. Die Erkenntnis der prognostischen Bedeutung der Herzinsuffizienz bei Diabetes geht einher mit einem besseren Verständnis der verschiedenen Herzinsuffizienzphänotypen mit reduzierter und erhaltener linksventrikulärer Funktion (HFrEF, HFpEF). Zusätzlich erlangte die Herzinsuffizienz bei Diabetes weitere Aufmerksamkeit durch kürzlich veröffentlichte klinische Studien, in denen gezeigt werden konnte, dass SGLT2-Inhibitoren wie Empagliflozin oder Dapagliflozin zu einer sehr raschen und hochsignifikanten Reduktion der Hospitalisierung für Herzinsuffizienz im Vergleich zu Placebo führten. Diese Daten haben den Fokus bei Diabetes von klassischen arte- rioskleroseassoziierten Endpunkten wie Myokardinfarkt und Schlaganfall umgelenkt auf klinisch mindestens ebenso bedeutsame Endpunkte wie Hospitalisierung für Herzinsuffizienz oder Tod durch Herzinsuffizienz.

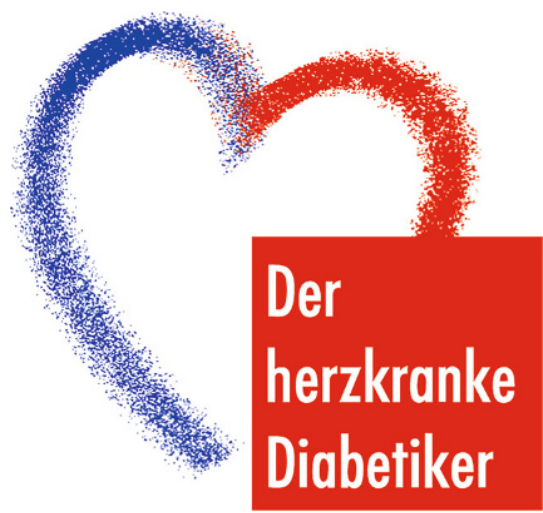

Stiftung in der

\section{Deutschen}

Diabetes-Stiftung

Für die Therapie der Herzinsuffizienz mit reduzierter Ejektionsfraktion (HFrEF) existieren sehr gute Daten zur Verbesserung der Prognose, aber für die - in der Hälfte der Fälle bei Patienten mit Diabetes vorliegende - Herzinsuffizienz mit erhaltener linksventrikulärer Funktion (HFpEF) besteht bislang nur eine symptomatische Therapie. Dies mag daran liegen, dass wir eine bessere Phänotypisierung der Herzinsuffizienzpatienten benötigen, um die Taxonomie der Herzkurden. Analysen großer Populationen 
insuffizienz $\mathrm{zu}$ verbessern. Hierzu ist es nötig, moderne Ansätze bioinformatischer Art, Clusteranalysen oder Big-data-Analysen einzusetzen, um die Herzinsuffizienz bei Patienten mit Diabetes besser $\mathrm{zu}$ charakterisieren und, basierend auf dieser besseren Charakterisierung, eine individualisierte und auf den Patienten zugeschnittene Therapie zu entwickeln.

Das vorliegende Heft „Herz und Diabetes" beleuchtet in verschiedenen Artikeln das Problem der Herzinsuffizienz bei Diabetes und adressiert therapeutische Strategien und neue Therapieansätze, das Risiko dieser Hochrisikopatienten zu modulieren. In jedem Fall benötigt es interdisziplinäre Ansätze zur Behandlung dieser Patienten. Dies ist auch das zentrale Anliegen der Stiftung „Der herzkanke Diabetiker“ in der Deutschen Diabetes-Stiftung, die in diesem Jahr ihr 20-jähriges Bestehen seit Gründung im Jahr 1999 begeht. Das vorliegende Heft ist diesem Jubiläum gewidmet und trägt dem dergestalt Rechnung, dass die Artikel in enger Abstimmung zwischen Kardiologen und Diabetologen geschrieben wurden.

\section{Korrespondenzadresse}

\section{Univ.-Prof. Dr. med. N. Marx}

Medizinische Klinik I - Kardiologie,

Angiologie und Internistische Intensivmedizin, Universitätsklinikum Aachen, RWTH Aachen

Pauwelsstraße 30, 52074 Aachen, Deutschland nmarx@ukaachen.de

Interessenkonflikt. N. Marx hat Vorträge gehalten für Boehringer Ingelheim, MSD, BMS, AstraZeneca, Lilly, NovoNordisk; N. Marx hat Forschungsprojekte durchgeführt, die von Boehringer Ingelheim und MSD unterstützt wurden, und als Berater für Boehringer Ingelheim, MSD, BMS, AstraZeneca, NovoNordisk fungiert. Sämtliche Honorare sind an die Uniklinik Aachen gegangen und N. Marx hat für seine Tätigkeit keine persönlichen Honorare erhalten. Darüber hinaus hat die Uniklinik Aachen Honorare für die Leitung klinischer Studien von Boehringer Ingelheim und NovoNordisk erhalten. R. Dörr und D. Tschöpe geben an, dass kein Interessenkonflikt besteht.

\section{Diabetes in der Schwanger- schaft}

\section{Das Risiko wird unterschätzt}

In Sachen Glukosestoffwechsel liegen in der Schwangerschaft Schutz und Schaden ganz eng beieinander. Auf der einen Seite sorgen Hormone der Plazenta in der zweiten Hälfte der Schwangerschaft dafür, dass der Blutzucker der Mutter nie zu tief absinkt, damit dem Ungeborenen über die Nabelschnur immer genug Energie zur Verfügung steht. Auf der anderen Seite kann dieses System auch entgleisen, so dass der Blutzucker nach jeder Mahlzeit, jedem Snack und jedem Glas Saft zu lange erhöht bleibt und das Kind immer wieder Phasen der Überernährung ausgesetzt ist. "Jede siebte Schwangere in Deutschland ist betroffen," erläutert Dr. med. Christian Albring, Präsident des Berufsverbandes der Frauenärzte.

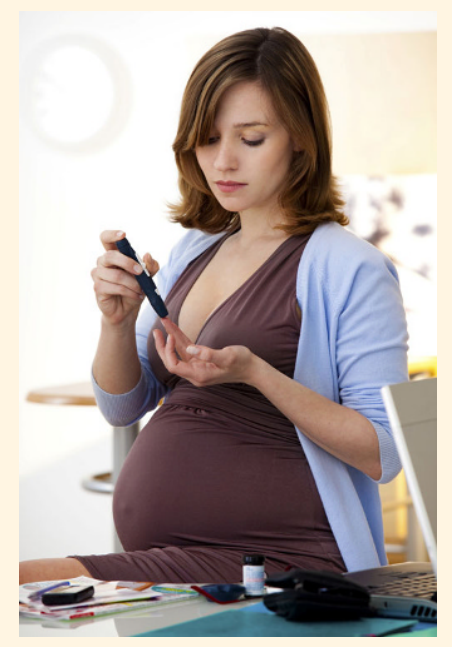

(c) RFBSIP / stock.adobe.com

Da der Gestationsbedingte Diabetes mellitus (GDM) das Risiko für viele Erkrankungen bei Mutter und Kind erhöht und zudem meist sehr gut behandelbar ist, sieht die gesetzliche Schwangerenvorsorge zwischen der 24. und 28. Woche ein GDM-Test vor. „Er sollte unbedingt wahrgenommen werden", so Albring. „Denn GDM ist zwar häufiger bei Frauen, die schon vor der Schwangerschaft übergewichtig waren. Er entwickelt sich aber genauso bei schlanken Frauen." Wichtig ist dann eine Ernährung ohne Süßes, aber stattdessen mit Nahrungsmitteln, die den Blutzucker weniger stark ansteigen lassen.
Oft stellt sich heraus, dass die Frauen sich doch reichlicher ernährt haben als es für sie und ihr Baby notwendig ist.

\section{Großer Kopf - schwierige Geburt}

Die häufigste Folge einer Überernährung ist, dass das Baby sehr groß ist und einen großen Kopfdurchmesser hat. Dann besteht die Gefahr, dass die natürliche Geburt den Beckenboden der Frau erheblich in Mitleidenschaft zieht. „In der Geburtshilfe gilt die Regel, wenn der Muttermund vollständig eröffnet ist und die Austreibungsphase mit den Presswehen beginnt, erhöht sich das Risiko für einen Riss oder Abriss der Beckenboden-Muskulatur in jeder Viertelstunde, die diese Phase dauert, um $8 \%$. Besonders Frauen über 35 Jahren sind gefährdet. Unwillkürlicher Wasserabgang und fehlende Kontrolle des Stuhlgangs können zu den Folgen gehören.

\section{Gegensteuern - mit richtiger Ernäh- rung und Bewegung}

Ist der Schwangerschaftsdiabetes erkannt, kann praktisch immer ein frühzeitiges energisches Gegensteuern diese Komplikationen vermeiden. Dazu muss die Schwangere ihre Ernährung sehr entschieden umstellen und viel Bewegung in ihren Alltag einbauen. In den meisten Fällen ist das bereits ausreichend, um den Blutzucker zu senken. „Leider gelingt das nicht immer", bedauert Albring. Manchmal hilft nur noch das regelmäßige Spritzen von Insulin bis zur Geburt.

\section{Kinder gehen belastet ins Leben} Kinder, deren Mütter einen unbehandelten Schwangerschaftsdiabetes in der Anamnese hatten, neigen selbst von Anfang an zu Übergewicht und haben später ein erhöhtes Risiko, einen Bluthochdruck und Diabetes zu entwickeln. Auch die Mütter selbst haben ein erhöhtes Risiko, später einen bleibenden Diabetes zu entwickeln. „Schwangerschaftsdiabetes ist keine Bagatelle. Wir sind froh, dass wir heute die Möglichkeit haben, den Test bei jeder Schwangeren im Rahmen der Mutterschaftsvorsorge durchzuführen."

Quelle: Berufsverband der Frauenärzte (BVF), www.bvf.de 


\section{Jahre Stiftung "Der herzkranke Diabetiker"}

Engagement für eine bessere Patientenversorgung

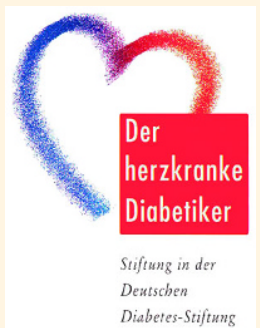

signifikant reduzieren lassen. Spätestens jetzt wurde gerade der Diabetiker auch zu einem kardiovaskulären Interventionspatienten. Allerdings war die klinische Datenlage den am Versorgungsprozess beteiligten Ärzten zum damaligen Zeitpunkt wenig bewusst und die Patienten mit Diabetes blieben hinsichtlich ihrer Endpunktprognose im Unklaren. Insofern kann es rückblickend als weiser Stiftungsauftrag betrachtet werden, genau diese Situation aufzugreifen und zu verbessern.

Die Stiftung DHD hat in der Folge vierzehn Mal einen jährlichen Förderpreis vergeben, der herausragende grundlagenwissenschaftliche- aber auch Versorgungsarbeiten an der Schnittstelle zwischen Herz- und Gefäßmedizin gewürdigt hat. Damit wurde ein wichtiger Beitrag geleistet, die Thematik Diabetes und Herz wissenschaftlich zu verankern, was angesichts der heute vorliegenden überwältigen Daten zur protektiven Wirkung neuer antidiabetischer Wirkstoffe auf die kardiovaskuläre Prognose dringend gebraucht wird. So sind in der aktuellen Stellungnahme der ESC zu Diabetes und Herzinsuffizienz allein drei der Autoren Stiftungspreisträger.

Unzählige Veröffentlichungen und Projekte zur allgemeinen Risikoaufklärung, u. a. mit der DHS haben zu notwendigerweise neuen Behandlungsalgorithmen, wie sie im aktuellen ADA-EASD Konsensuspapier formuliert sind, beigetragen. Man kann sagen, die Stiftung hat den "herzkranken Diabetiker" als eigene Behandlungsentität eingeführt. Ausdrücklich nicht vergessen sind dabei die aufs engste verbundenen anderen vaskulären Modalitäten, etwa im Bereich der Neurologie, der Angiologie und der Nephrologie, wie sie auch im aktuell tätigen Kuratorium repräsentiert sind.

Die Stiftung hat auf allen Ebenen der Medizinund Wissenschaftspublizistik die Thematik in die Breite getragen. Die elektronischen Medien haben dabei eine wichtige Rolle gespielt.
So wurde gerade 2018 ein Relaunch der Webrepräsentanz durchgeführt, der Geschichte und Leistungen wiedergibt. Mit einem eigenen Curriculum "Herz- und Diabetes" in Kooperation mit DGK und DDG unter dem Dach der Akademie für medizinische Fortbildung der Ärztekammer Westfalen-Lippe bietet die Stiftung die relevanten Themeninhalte als standardisierte Fortbildung mit Präsenz und E-Learning ab 2019 an. Es ist zu wünschen, dass die Stiftung DHD auch weiterhin so aktiv hilft, die Dynamik der Erkenntnisse voranzubringen und in eine entsprechende Anpassung der Versorgungsalgorithmen zu übersetzen.

Dies wird nur durch Engagement, Neutralität und Wissenschaftsbasierung möglich sein. Immerhin ist entlang der Stiftungsarbeit über die zurückliegenden 20 Jahre aus einem einfachen Vorschlag ein breit akzeptiertes Versorgungsmodell mit multiplen Angeboten von der Prävention über die Pharmakotherapie bishin zur Intervention geworden.

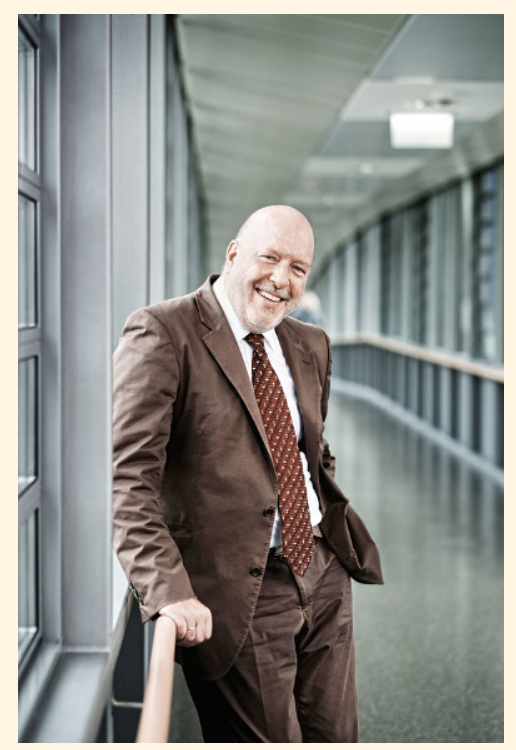

Univ.-Prof. Dr. med. Dr. h. c. D. Tschöpe Vorsitzender der Stiftung DHD 